\title{
Cannabinoid-associated cell death mechanisms in tumor models (Review)
}

\author{
GIUSEPPE CALVARUSO, ORNELLA PELLERITO, ANTONIETTA NOTARO and MICHELA GIULIANO
}

Department of Experimental Biomedicine and Clinical Neuroscience, Section of Biochemical

Sciences, University of Palermo, 90129 Palermo, Italy

Received January 25, 2012; Accepted March 9, 2012

DOI: 10.3892/ijo.2012.1476

\begin{abstract}
In recent years, cannabinoids (the active components of Cannabis sativa) and their derivatives have received considerable interest due to findings that they can affect the viability and invasiveness of a variety of different cancer cells. Moreover, in addition to their inhibitory effects on tumor growth and migration, angiogenesis and metastasis, the ability of these compounds to induce different pathways of cell death has been highlighted. Here, we review the most recent results generating interest in the field of death mechanisms induced by cannabinoids in cancer cells. In particular, we analyze the pathways triggered by cannabinoids to induce apoptosis or autophagy and investigate the interplay between the two processes. Overall, the results reported here suggest that the exploration of molecular mechanisms induced by cannabinoids in cancer cells can contribute to the development of safe and effective treatments in cancer therapy.
\end{abstract}

\section{Contents}

1. Introduction

2. Cannabinoids

3. Cannabinoid-induced apoptotic pathways

4. Cannabinoid-induced autophagic pathways

5. Synergistic effects of cannabinoids in combination with other drugs

6. Conclusion

\section{Introduction}

The health and survival of every living organism is based on the crucial balance between cellular life and death pathways.

Correspondence to: Professor Michela Giuliano, Department of Experimental Biomedicine and Clinical Neuroscience, Section of Biochemical Sciences, University of Palermo, Via del Vespro 127, 90129 Palermo, Italy

E-mail: michela.giuliano@unipa.it

Key words: cannabinoids, apoptosis, autophagy, synergistic effects
To maintain a normal size, each tissue must balance the plethora of opposite signals which arrive on its membrane surface and finely control each to prevent pathological conditions such as cancer.

For many decades, the molecular bases of cancer transformation were related to the gain of function of oncogenes or the inactivation of tumor suppressor genes, two classes of genes involved in regulating cell proliferation $(1,2)$. More recently, this classification has been extended when it became clear that neoplastic transformation is related not only to the uncontrolled proliferative thrust but also to the loss of death ability $(3,4)$. Stimulation and/or restoration of cell death can lead to the suppression of transformation and tumorigenesis (4). From then on, the list of tumor suppressors with death-inducing action towards tumor cells has lengthened progressively. Moreover, mutations which affect specific genes controlling death signalling can compromise the effectiveness of chemotherapy (5).

Recent studies have shown that tumor cells can activate different pathways of death, such as apoptosis, necrosis, mitotic catastrophe and autophagy which very often are activated by the same oncogenic factors related to proliferative signals (6). Signalling molecules and pathways involved in these alternative forms of cell death have not yet been defined and the activation of a specific cell death mechanism may depend on the metabolic context, the genotype and/or phenotype of the cell, or the kind of damage. Thus, the relative contribution of these different modes of cell death remains to be defined.

Apoptosis, the first identified process of genetically programmed cell death, has been extensively studied and its contribution to the pathogenesis of cancer is well documented. Classical studies on apoptotic pathways evidenced the activation of caspase family proteases as a key step in apoptotic cell death (7). Caspases can be initiated through the extrinsic (receptor) pathway or at the mitochondria by stimulating the intrinsic pathway $(8,9)$ and following the activation they are responsible for the cleavage of a number of different substrates leading to many of the morphologic features of apoptotic cell death.

Another interesting event in cancer is the balance between the expression of survival factors and the induction of apoptotic ones. It is well known that cancer cells show constitutive activation of survival factors which sustain deregulated cell proliferation and counteract death stimuli (10). The survival factors which are very often related to tumorigenicity are 
survivin, a member of inhibitor of apoptosis protein (IAP) family and AKT. Survivin sustains cell survival maintaining inactivated the executioner caspases (11). The other survival factor, the serine/threonine protein kinase AKT, is widely recognised as a key mediator of growth factor-promoted cell survival. It sustains nutrient uptake, macromolecular synthesis and ATP production. Also, this factor which is constitutively active in many types of human cancer (12), plays an anti-apoptotic role in the cell. In fact, it phosphorylates and inactivates some members of Bcl-2 and caspase families.

Surprisingly, a new mechanism of cell death in cancer cells has been demonstrated, which has been related to the activation of autophagic pathway $(13,14)$. Originally known as a housekeeping process responsible for the protein and organelle turnover, autophagy has also been indicated to play a significant role in cell death.

Autophagic process is achieved through two pathways: microautophagy and macroautophagy, which can be further classified into canonical and non-canonical forms (14). They differ in the transport of the material to be degraded within the lysosomal lumen, the type of transported material and its regulation. Microautophagy has been traditionally regarded as a form of active autophagy to ensure the turnover of longlived proteins in basal conditions (15). During microautophagy, lysosomes incorporate and digest regions of cytosol, including proteins and cytoplasmic organelles, by invagination or protrusion without the intermediate formation of autophagic vacuoles.

Macroautophagy (or autophagy) is responsible for the degradation of soluble proteins and organelles under stress conditions (16). It involves the formation of double-membrane vacuoles (autophagosomes) that sequester portions of the cytoplasm and translocate them to the lysosomes (17).

To date, $>30$ autophagy-related genes (ATG) required for autophagy and their related pathways have been identified and analysed as markers of the different steps of autophagic process. Some, such as Atg1, are involved in upstream steps of autophagosome formation; others, such as Beclin-1 (Atg6), perform their role as part of a core complex that contains vacuolar sorting protein 34 (VPS34); others, such as LC-3, a protein which is lipidated and inserted into the outer and inner surface of autophagosomes, are essential for the biogenesis of the large double-membrane vesicles (18). Once digested by autophagosome, the substances are reabsorbed in the vacuole and made available to the cells.

At the molecular level, autophagy is regulated by many protein factors which are involved in key steps of the mechanism. The PI3K/AKT/mTOR axis and AMP kinase are the two major pathways involved in autophagy induction. mTOR is a repressor of autophagy because it is responsible for Atg1 hyper-phosphorylation, an event that inhibits the formation of induction complex. A correlation between AKT and mTOR pathway exists; in fact, AKT is able to positively regulate mTOR since it phosphorylates and inhibits TSC1/TSC2 complex which is, in turn, responsible for mTOR inactivation. This inhibition allows mTOR to act freely thus inhibiting autophagy (19).

The other crucial pathway involved in autophagy is sustained by AMPK. This protein is activated by an increase in the intracellular AMP/ATP ratio as a consequence of energy deficit. This event is responsible for mTOR inhibition and p53 phosphorylation, inducing increase in autophagy and arrest of cell cycle progression, respectively (20).

In an attempt to explain the exact role of autophagy in cancer process, Eisenberg-Lerner and Kimchi (21) recently presented a model in which the role of autophagy is dependent on the stage of cell life, i.e. precancerous stage, transformed cell or solid tumor. In the first two stages autophagy could accelerate tumor development whereas in solid tumors, when apoptosis is absent, autophagy program could be antitumorigenic.

It has been demonstrated that autophagy can undertake a complex interplay with apoptosis (22). In relation to cell type, it could serve as a cell survival pathway suppressing apoptosis, or in others, it could trigger a death pathway either in collaboration with apoptosis or substituting to that when the former is defective. It is very difficult to distinguish the two pathways because some death stimuli can activate both of them and which pathway will be undertaken depends on the molecular expression profile of the cell type (23). An interesting observation concerns the role explained by ER-stress response in determining cell fate. Different injuries, such as calcium imbalance, oxidative stress or misfolded proteins, trigger a condition indicated as stress of endoplasmic reticulum (ER-stress) which can lead to different cell responses. ER-stress can drive mitochondria-dependent apoptosis when there is the activation of the transcription factor CHOP; or it can determine cell survival when it is accompanied by the increase in the level of GRP78, the main intraluminar HSP70 that protects cells from the injury. Differently, if the level of the CHOP target TRB-3 significantly increases, ER-stress triggers autophagy.

The cross-talk between apoptosis and autophagy is therefore quite complex, and sometimes contradictory, but surely critical to the overall cell fate.

\section{Cannabinoids}

Cannabis derivatives (cannabinoids) include the bioactive constituents of cannabis $\left(\Delta^{9}\right.$-tetrahydrocannabinol, cannabidiol), endogenous lipids with cannabinoid-like activity (anandamide, 2-arachydonoyl-glycerol) and the synthetic analogues (WIN55,212-2, methanandamide, the stable analogue of anandamide, JWH-015, HU-210, HU-331) (24).

In the last few years, investigations have suggested the potential application of cannabinoids as antitumor drugs because in many in vivo and in vitro tumor models the activation of the cannabinoid system induces cell cycle arrest, inhibition of cell survival and activation of programmed cell death signalling (25). Apart from regulating tumor cell growth and death, other antitumorigenic mechanisms of cannabinoids are currently emerging as a focus of research work, such as their effects on tumor neovascularization, cell migration, adhesion, invasion and metastasis (26). The present review focuses on the impact of cannabinoids on apoptosis and autophagy, the two main death pathways responsible for cancer cell death.

\section{Cannabinoid-induced apoptotic pathways}

Although the antitumor properties of cannabinoids were first observed more than 30 years ago, when Munson et al (27) demonstrated that $\Delta^{9}$-tetrahydrocannabinoid (THC) inhibits lung adenocarcinoma cell growth in vivo, the elucidation of 
mechanisms employed by cannabinoids for influencing cancer cell proliferation and death was developed in the last two decades. However, there are still many obscure sides on death pathways activated by these compounds and, in particular, on the different contribution of apoptosis and autophagy in cell death.

The anticancer potential of this class of compounds can be very different in the various tumor systems. This depends on the mechanism used by cannabinoids to interact with the cells, i.e. the class of receptors to which they bind or on the specific intracellular activated pathways.

Regarding the interaction with the target cell, it has been demonstrated that cannabinoids can interact with the specific type 1 or 2 cannabinoid receptors (CB1 and $\mathrm{CB} 2$ ), which are differently expressed in neural and peripheral tissues, respectively, with transient receptor potential channels of the vanilloid type-1 (TRPV1), or directly with membrane microdomains rich in cholesterol named lipid rafts (28). The interaction of cannabinoids with the different receptor types often leads to the same cell fate even if different intracellular signalling cascades can be activated. For example, the activation of $\mathrm{CB}$ receptors can be responsible for the increase in the level of ceramide or the decrease of cAMP level via inhibition of adenilate cyclase. These events result in canonical apoptosis mediated by the activation of caspase activities $(29,30)$. It has also been demonstrated that the interaction of cannabinoids with TRPV receptors causes the activation of overlapping mechanisms, such as the mitochondrial apoptotic pathway, although in this case the event is accompanied by the increase in the level of ROS with the consequent oxidative stress (31). However, sometimes the effects of cannabinoids can not be counteracted by the addition of selective cannabinoid receptor antagonists, hypothesizing that another type of interaction between cannabinoids and cell membrane can be present. In hepatic stellate cells, an interaction between anandamide and lipid rafts, the membrane micro-domains rich in cholesterol, was found. This relationship induces downregulation of PI3K/ AKT signalling pathway with a possible role in inflammatory process (32). Differently, in hepatocellular carcinoma cells, as well as in melanoma cells, the interaction of the synthetic cannabinoid WIN55,212-2 to lipid rafts is able to promote a canonical caspase-dependent apoptotic pathway $(33,34)$. Moreover, it is not possible to exclude that the activation of $\mathrm{CB} 1$ receptors and the consequent induction of cannabinoid signalling could be mediated by the association of receptor with lipid rafts, as recently demonstrated by Oddi et al (35) in elegant experiments of site-specific mutations of palmitoylated cysteine residue of $\mathrm{CB} 1$ receptors.

An exception to that previously reported is represented by GPR55, a novel potential cannabinoid receptor, whose activation is strictly related to cell proliferation (36).

The analysis of intracellular mediators of apoptotic cell death induced by cannabinoids leads to the identification of some molecules, such as ceramide, reactive oxygen species (ROS), mitogenic kinases (MAPK) and some survival factors, that, more than others, seem to be representative of cannabinoid actions. Ceramide is one of the first molecules identified as a mediator of cannabinoid action. The induction of ceramide accumulation mediated by $\mathrm{CB}$ receptors leads to apoptosis in mantle cell lymphoma, glioma, colon and pancreatic cancer
(37-40). The increase in ceramide level can be dependent on the de novo synthesis or the release from membrane sphyngolipids induced by the activation of sphyngomyelinase. The different origins of ceramide can be disclosed by the employment of specific inhibitors of the two enzymes, fumonisin B1, the inhibitor of ceramide synthase, or desipramine, an inhibitor of sphyngomyelinase $(41,42)$.

In many cannabinoid signalling pathways, ROS can exert a crucial role in activating both initiator and executioner caspase activities suggesting that continuous oxidative stress can occur following cannabinoid exposure. The involvement of ROS in cannabinoid-induced apoptosis has been widely evidenced in glioma and leukemia cells $(43,44)$. Moreover, the demonstration of ROS involvement in cannabinoid-induced apoptosis is also confirmed by the employement of $\mathrm{N}$-acetyl-cysteine, a thiol antioxidant that scavenges ROS, or the NAD(P)H oxidase inhibitors, which are able to attenuate cannabinoid effects. A strong interplay between ceramide and generation of oxidative stress has been demonstrated. Ceramide stimulates the formation of ROS and apoptotic mechanisms, establishing a link between sphingolipid metabolism and oxidative stress. Moreover, inhibition of ROS generating enzymes or treatment with antioxidants impairs sphingomyelinase activation and ceramide production. Therefore, it is plausible to hypothesize that the contemporaneous activation of CB and TRP vanilloid receptors, responsible for the triggering of the two intracellular mediators (ceramide and ROS), can augment the effects induced by cannabinoids carrying out an amplification of caspase cascades.

The study on downstream events following ROS generation or ceramide induction has evidenced the involvement of ER-stress. In many experimental tumor models, high ROS levels induce ER-stress as demonstrated by the increase in the level of specific ER-stress mediators (p8, CHOP, TRB-3 and GRP-78) which, in turn, are responsible for the activation of mitochondrial intrinsic apoptotic pathway (45). On the other hand, a relationship between the increase in ceramide level and ER-stress is also well documented in cannabinoid-induced apoptosis in tumor cells (46). The activation of p8/CHOP axis in cannabinoid-induced apoptosis has also been documented $(40,47)$.

Another pathway, which has been demonstrated to be modulated following cannabinoid exposure, is represented by mitogen-activated protein kinase (ERK, JNK and p38/MAPK) cascades. The main players of these pathways are a group of serine/threonine protein kinases that convert extracellular stress stimuli into different, sometimes opposite, cellular responses, including cell cycle arrest, apoptotic cell death and cytokine production, through the phosphorylation of specific targets. A plethora of data reports the activation of stress-activated protein kinases or extracellular-related signal kinases in cannabinoid-dependent control of cancer cell growth and survival $(43,48)$. Interestingly, it has been demonstrated that the duration of the stimulus can be fundamental for the type of cellular response. A transient activation of ERK cascade leads to cell survival and proliferation, while long-term ERK activation results to apoptotic response as proposed by GalveRoperh et al (48) in malignant glioma and confirmed in Kaposi sarcoma cells (49). In this cell model, WIN55,212-2 induced phosphorylation of ERK $1 / 2$ at the beginning of its action. Then, the noxious stimulus may trigger activation of stress kinases 
JNK and p38 which, in turn, activate downstream mechanisms leading Kaposi's sarcoma cells to apoptotic death (49). The activation of ERK 1/2 has been recently demonstrated also in gastric cancer; in this case, it is related to cell cycle arrest in G0/G1 phase of proliferative cycle, an event which precedes apoptotic response (50). Differently, studies on several prostate and ovarian cancer cell lines evidenced that the activation of ERK signalling by the putative cannabinoid receptor GPR55 activates an autocrine loop that sustains cell proliferation (51).

It is well known that the activation of death pathways needs to be accompanied by downregulation of survival factors. A central intracellular pro-survival signalling is represented by the PI3K/AKT pathway, whose importance in different cancers is also corroborated by clinical studies. This kinase is the central node of PI3K/AKT/mTOR signalling pathway that activates crucial processes such as cell survival, growth, proliferation, angiogenesis, and cell migration and invasion (52). On the contrary, inhibition of pAKT leads to cell cycle arrest which precedes apoptotic response very often mediated by the involvement of intrinsic mitochondria-dependent pathway. Downregulation of AKT is involved in cannabinoid antitumoral action. In human gastric cancer cells, it has been reported that cannabinoid receptor agonists induce cell cycle arrest which is a consequence of AKT inhibition related to the activation of MAPK pathway (50). Interesting results were also obtained in astrocytoma (53). In this model, cannabinoids induce apoptosis only in cells expressing low levels of $\mathrm{CB}$ receptors, while in cells with high $\mathrm{CB}$ receptor levels, cannabinoids are ineffective because of the contemporaneous high amount of the phosphorylated pro-survival AKT. These results suggest that the coupling of $\mathrm{CB}$ receptors to AKT pathway (when these receptors are expressed at mid and high levels) eliminates the ability of cannabinoids to induce apoptosis in astrocytoma cells. Therefore, AKT pathway is an attractive target for anticancer agents and clinical trials with PI3K or AKT inhibitors could reserve encouraging results.

\section{Cannabinoid-induced autophagic pathways}

As previously indicated, autophagy can assume different and opposite roles in cell fate. In fact, this process may trigger survival pathways, collaborate with apoptosis to induce cell death or substitute itself to apoptosis to start an autonomous death pathway.

Numerous recent studies have indicated the cannabinoidmediated induction of autophagy in different experimental cancer models. Noteworthy is the observation that the activation of autophagic pathway is often mediated by the same molecules that are involved in apoptosis.

In human glioma cells, the main cancer model for studying cannabinoid action, it has been observed that $\Delta^{9}$-tetrahydrocannabinol (THC) induces cell death through stimulation of autophagy (54). Data indicate that THC induces ceramide accumulation, phosphorylation of elF $2 \alpha$ and ER stress which activates autophagy via TRB3-dependent inhibition of the AKT/mTOR axis. In these cells autophagy seems to be upstream of apoptosis in cannabinoid-induced cancer cell death. The stimulation of autophagy-mediated apoptosis induced by cannabinoids has also been demonstrated in animal models of cancer, including glioma $(54,55)$. Similar studies performed to investigate the effects of cannabinoids on the growth of hepatocellular carcinoma cells have demonstrated that THC markedly reduces the viability of the human hepatocellular carcinoma cell lines through induction of autophagy mediated, also in this case, by upregulation of TRB3 and subsequent inhibition of the serine-threonine kinase AKT and AMPK stimulation. As in glioma animal model, in vivo studies on hepatocellular carcinoma subcutaneous xenografts have revealed that THC-dependent growth inhibition is reduced when autophagy is genetically or pharmacologically blocked, thus confirming that the induction of autophagy can be a key step in cannabinoid-induced cell death (56).

Further biochemical pathways have been suggested in the attempt to clarify the molecular mechanisms of cell death induced by cannabinoids. In breast cancer cells, cannabidiol induces ER-stress and subsequently inhibition of AKT and mTOR signalling, indicating autophagy activation. In addition, data have also shown the activation of intrinsic apoptotic pathway. The coexistence of autophagy and apoptosis has been confirmed by different observations: the inhibition of cannabidiol-dependent ROS production blocked the induction of both processes and the increase in the level of beclin-1, a marker of autophagy, plays a central role in the induction of cannabidiol-mediated apoptosis (57).

A new recent study on the interplay between autophagy and apoptosis induced by cannabinoids showed a surprise in mantle cell lymphomas (58). Interestingly, this study shows that the response to cannabinoid treatment decreases cell viability which does not involve the caspase- 3 cascade. Moreover, mantle cell lymphoma primary cells respond to cannabinoid treatment through the formation of cycloheximide-sensitive cytoplasmic vacuoles. However, the lack of enhanced autophagosome formation and lysosomal contribution also excludes the involvement of a canonical autophagic process. The authors hypothesize that the observed features resemble paraptosis-like cell death, a third type of programmed cell death, not previously described in response to cannabinoids. Activation of more types of cell death by cannabinoids widens their potential therapeutic usefulness in cells overexpressing cannabinoid receptors.

\section{Synergistic effects of cannabinoids in combination with other drugs}

Recent studies have demonstrated the ability of cannabinoids to synergize with other molecules to trigger death pathways in cancer cells. It is well known that in cancer therapy the employment of combinations of drugs rather than a single drug represents a therapeutic strategy with distinct advantages. On the one hand, the contemporaneous activation of different biochemical pathways can achieve synergistic effects; on the other hand, the combination can result in a reduction of the dose of each single drug thereby reducing side effects. Thus, the ability of cannabinoids in synergizing with other drugs to improve their anticancer activity has been investigated. In particular, we have recently demonstrated that the synthetic cannabinoid WIN55,212-2 sensitizes hepatocellular carcinoma cells to apoptosis, mediated by tumor necrosis-related apoptosis inducing ligand (TRAIL). The molecular mechanism induced by WIN/TRAIL combined treatment, involves activation of the extrinsic apoptotic pathway through upregulation of TRAIL 


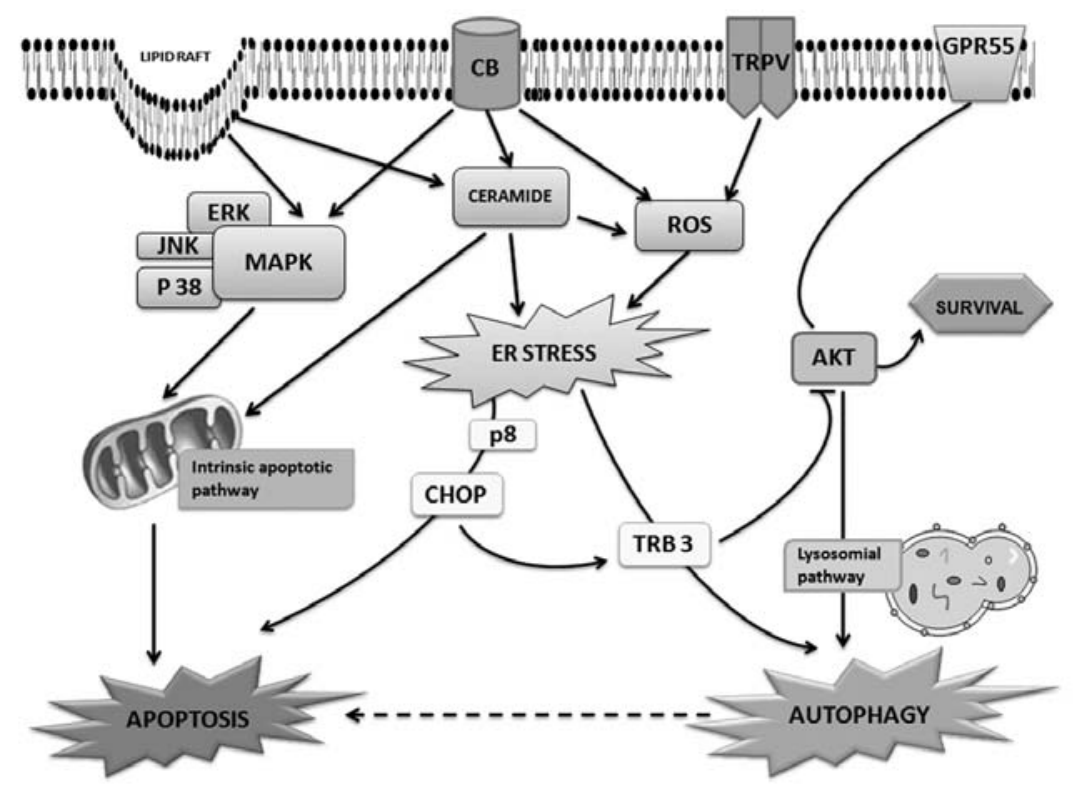

Figure 1. Schematic representation of the cannabinoid-induced cell death pathways.

death receptor DR5 (47). This event seems to be related to the increase in the level of p8 and CHOP, two factors implicated in ER stress response and apoptosis. Moreover, WIN55,212-2 treatment also induces a marked downregulation of some survival factors. Therefore, both the induction of DR5 and the decrease of survival factors explain synergistic effects of the drugs in hepatocellular carcinoma cells. Our unpublished data obtained in osteosarcoma cells seem to indicate that WIN also triggers an autophagic pathway with the increase in the level of beclin-1 and LC-3 II, but this pathway is not carried out because of the lysosomal membrane permeabilization. Also, in pancreatic cancer cells the combination of cannabinoids with gemcitabine, a pyrimidine analog largely employed in anticancer therapy, induces synergistic effects via activation of autophagy (59). In this case, gemcitabine induces upregulation of both $\mathrm{CB}$ receptors thus sensitizing cells to cannabinoid effects. A central role in this pathway seems to be related to the increase in ROS production, induction of ER-stress which carried out to specific cell death pathway of type II (autophagy). A curious observation about the therapeutic potential of cannabinoids is that in the first example cannabinoids sensitize the cells to TRAIL-induced cell death while in the latter the cannabinoid death action is potentiated by gemcitabine addition in pancreatic cancer cells. Another example of synergistic effects of cannabinoids with other drugs has also been reported by Gustafsson et al (60) who demonstrated that the synthetic cannabinoid HU210, anandamide and its other derivatives induce synergistic and cytotoxic, rather than antiproliferative, effects when employed in combination with the classic pyrimidine antagonist 5-fluorouracil (5-FU) in the colorectal carcinoma cells. The authors report that the effect does not seem to involve cannabinoid receptors and suggest the involvement of ER-stress because the employment of common antioxidants attenuates cannabinoid cytotoxicity.

Autophagy is also involved in the strong antitumoral effects induced in glioma xenografts by combined adminis- tration of THC and temozolomide, the benchmark agent for the management of glioblastoma multiforme, an effect that is also observed in tumors which are resistant to temozolomide treatment. THC/temozolomide combined treatment enhances autophagy, whereas pharmacologic or genetic inhibition of this process prevents the effects of combined treatment, supporting that the activation of autophagy plays a crucial role in the mechanism of action of this drug combination (55).

\section{Conclusion}

Data reported in this review seem to confirm the ability of cannabinoids to induce cell death in different tumor models. Moreover, it can be seen from the brief literature overview presented here that these compounds are effective in inducing the main cell death modes, i.e. apoptosis and autophagy, and that cell fate will depend on the role and the interplay among these different death signals. Unfortunately, to simplify the mechanisms induced by cannabinoids to carry out cells to death and discriminate the role played by each intracellular mediator appears to be very difficult for different reasons: i) the two pathways often employ the same molecules (Fig. 1) and ii) the identification of autophagy as cell death program of type II is very recent as well as the discovery that many apoptotic pathways are preceded by the activation of autophagy. Therefore, it is not possible to exclude that a cannabinoid-triggered death pathway which was initially identified as a canonical apoptotic cell death hides a 'secret step of autophagic activation'. Furthermore, the observation that cannabinoids can synergize with other molecules thus accelerating death pathway, make these compounds, employed alone or in combination, promising for clinical outcome.

\section{References}

1. Weinberg RA: The molecular basis of oncogenes and tumor suppressor genes. Ann NY Acad Sci 758: 331-338, 1995. 
2. Yuspa SH, Długosz AA, Cheng CK, Denning MF, Tennenbaum T, Glick AB and Weinberg WC: Role of oncogenes and tumor suppressor genes in multistage carcinogenesis. J Invest Dermatol 103: S90-S95, 1994.

3. Lowe SW and Lin AW: Apoptosis in cancer. Carcinogenesis 21: 485-495, 2000

4. Brown JM and Attardi LD: The role of apoptosis in cancer development and treatment response. Nat Rev Cancer 5 : 231-237, 2005

5. Brown JM and Wilson G: Apoptosis genes and resistance to cancer therapy: what does the experimental and clinical data tell us? Cancer Biol Ther 2: 477-490, 2003.

6. Tsatsanis $C$ and Spandidos DA: Oncogenic kinases signaling in human neoplasms. Ann NY Acad Sci 1028: 168-175, 2004.

7. Degterev A, Boyce M and Yuan J: A decade of caspases. Oncogene 22: 8543-8567, 2003.

8. Peter ME and Krammer PH: The CD95(APO-1/Fas) DISC and beyond. Cell Death Differ 10: 26-35, 2003.

9. Green DR and Kroemer G: The pathophysiology of mitochondrial cell death. Science 305: 626-629, 2004.

10. Fassetta M, D'Alessandro L, Coltella N, Di Renzo MF and Rasola A: Hepatocyte growth factor installs a survival platform for colorectal cancer cell invasive growth and overcomes p38 MAPK-mediated apoptosis. Cell Signal 18: 1967-1976, 2006.

11. Altieri DC: Survivin and IAP proteins in cell-death mechanisms. Biochem J 430: 199-205, 2010

12. Tokunaga E, Oki E, Egashira A, Sadanaga N, Morita M, Kakeji Y and Maehara Y: Deregulation of the AKT pathway in human cancer. Curr Cancer Drug Targets 8: 27-36, 2008.

13. Mathew R: Role of autophagy in cancer. Nat Rev Cancer 7 : 961-967, 2007.

14. Kimmelman AC: The dynamic nature of autophagy in cancer Genes Dev 25: 1999-2010, 2011.

15. Krick R, Muehe Y,Prick T,BremerS,Schlotterhose P,EskelinenEL, Millen J, Goldfarb DS and Thumm M: Microautophagy of the nucleus requires the core macroautophagy genes. Mol Biol Cell 19: 4492-4505, 2008.

16. Farré JC and Subramani S: Peroxisome turnover by micropexophagy: an autophagy-related process Cell Biol 14: 515-523, 2004.

17. Periyasamy-Thandavan S, Jiang M, Schoenlein P and Dong Z Autophagy: molecular machinery, regulation, and implications for renal pathophysiology. Am J Physiol Renal Physiol 297: F244-F256, 2009.

18. Mizushima N: The role of the Atg1/ULK1 complex in autophagy regulation. Curr Op Cell Biol 22: 132-139, 2010.

19. Sarbassov DD, Guertin DA, Ali SM and Sabatini DM: Phosphorylation and regulation of $\mathrm{AKT} / \mathrm{PKB}$ by the rictormTOR complex. Science 307: 1098-1101, 2005.

20. Hardie D: AMP-activated/SNF1 protein kinases: conserved guardians of cellular energy. Nat Rev Mol Cell Biol 8: 774-781, 2007.

21. Eisenberg-Lerner A and Kimchi A: The paradox of autophagy and its implication in cancer etiology and therapy. Apoptosis 14 376-391, 2009

22. Djavaheri-Mergny M, Maiuri MC and Kroemer G: Cross talk between apoptosis and autophagy by caspase-mediated cleavage of Beclin 1. Oncogene 29: 1717-1719, 2010.

23. Wong $\mathrm{CH}$, Iskandar $\mathrm{KB}$, Yadav SK Hirpara JL, Loh $\mathrm{T}$ and Pervaiz S: Simultaneous induction of non-canonical autophagy and apoptosis in cancer cells by ROS-dependent ERK and JNK activation. PLoS One 5: e9996, 2010.

24. Pertwee RG: Cannabinoid receptor ligands: clinical and neuropharmacological considerations, relevant to future drug discovery and development. Expt Op Invest Drugs 9: 1553$1571,2000$.

25. Guzmàn M: Cannabinoids: potential anticancer agents. Nat Rev Cancer 3: 745-755, 2003.

26. Freimuth N, Ramer R and Hinz B: Antitumorigenic effects of cannabinoids beyond apoptosis. J Pharmacol Exp Therapeutics 332: 336-344, 2010.

27. Munson AE, Harris LS, Friedman MA, Dewey WL and Carchman RA: Antineoplastic activity of cannabinoids. J Nat Cancer Inst 55: 597-602, 1975.

28. Demuth DG and Molleman A: Cannabinoid signalling. Life Sci 78: 549-563, 2006

29. Herrera B, Carracedo A, Diez-Zaera M, Gómez del Pulgar T, Guzmán M and Velasco G: The CB2 cannabinoid receptor signals apoptosis via ceramide-dependent activation of the mitochondrial intrinsic pathway. Exp Cell Res 312: 2121-2131, 2006.
30. Bifulco M,Malfitano AM,PisantiS andLaezzaC: Endocannabinoids in endocrine and related tumours. Endocr Relat Cancer 15: 391-408, 2008.

31. Maccarrone M, Lorenzon T, Bari M, Melino G and FinazziAgro A: Anandamide induces apoptosis in human cells via vanilloid receptors. Evidence for a protective role of cannabinoid receptors. J Biol Chem 275: 31938-31945, 2000

32. Yang Q, Liu HY, Zhang YW, Wu WJ and Tang WX: Anandamide induces cell death through lipid rafts in hepatic stellate cells. J Gastroenterol Hepatol 25: 991-1001, 2010.

33. Giuliano M, Pellerito O, Portanova P, Calvaruso G, Santulli A, De Blasio A, Vento R and Tesoriere G: Apoptosis induced in HepG2 cells by the synthetic cannabinoid WIN: involvement of the transcription factor PPARgamma. Biochimie 91: 457-465, 2009.

34. Scuderi MR, Cantarella G, Scollo M, Lempereur L, Palumbo M, Saccani-Jotti G and Bernardini R: The antimitogenic effect of the cannabinoid receptor agonist WIN55212-2 on human melanoma cells is mediated by the membrane lipid raft. Cancer Lett 310 : 240-249, 2011

35. Oddi S, Dainese E, Sandiford S, Fezza F, Lanuti M, Chiurchiù V, Totaro A, Catanzaro G, Barcaroli D, De Laurenzi V, et al: Palmitoylation of cysteine 415 of helix 8 : effect on membrane localisation and signalling of the $\mathrm{CB}(1)$ cannabinoid receptor. $\mathrm{Br}$ J Pharmacol 165: 2635-2651, 2011.

36. Hu G, Ren G and Shi Y: The putative cannabinoid receptor GPR55 promotes cancer cell proliferation. Oncogene 30: 139-141, 2011.

37. Gustafsson K, Christensson B, Sander B and Flygare J: Cannabinoid receptor-mediated apoptosis induced by $\mathrm{R}(+)$ methanandamide and Win55,212-2 is associated with ceramide accumulation and p38 activation in mantle cell lymphoma. Mol Pharmacol 70: 1612-1620, 2006.

38. Blazquez C,Carracedo A, Salazar M,Lorente M,Egia A, GonzalezFeria L, Haro A, Velasco G and Guzman M: Down-regulation of tissue inhibitor of metalloproteinases-1 in gliomas: a new marker of cannabinoid antitumoral activity? Neuropharmacology 54: 235-243, 2008

39. Cianchi F, Papucci L, Schiavone N, Lulli M, Magnelli L, Vinci MC, Messerini L, Manera C, Ronconi E, Romagnani P, et al: Cannabinoid receptor activation induces apoptosis through tumor necrosis factor alpha-mediated ceramide de novo synthesis in colon cancer cells. Clin Cancer Res 14: 7691-7700, 2008.

40. Carracedo A, Gironella M, Lorente M, Garcia S, Guzman M, Velasco G and Iovanna JL: Cannabinoids induce apoptosis of pancreatic tumor cells via endoplasmic reticulum stress-related genes. Cancer Res 66: 6748-6755, 2006.

41. Olea-Herrero N, Vara D, Malagarie-Cazenave S and DíazLaviada I: The cannabinoid R+ methanandamide induces IL-6 secretion by prostate cancer PC 3 cells. J Immunotoxicol 6 : 249-256, 2009.

42. Giuliano M, Calvaruso G, Pellerito O, Portanova P, Carlisi D, Vento R and Tesoriere G: Anandamide-induced apoptosis in Chang liver cells involves ceramide and JNK/AP-1 pathway. Int J Mol Med 17: 811-819, 2006.

43. Massi P, Vaccani A, Bianchessi S, Costa B, Macchi P and Parolaro D: The non-psychoactive cannabidiol triggers caspase activation and oxidative stress in human glioma cells. Cell Mol Life Sci 63: 2057-2066, 2006.

44. McKallip RJ, Jia W, Schlomer J, Warren JW, Nagarkatti PS and Nagarkatti M: Cannabidiol-induced apoptosis in human leukemia cells: a novel role of cannabidiol in the regulation of p22phox and Nox4 expression. Mol Pharmacol 70: 897-908, 2006.

45. Malhotra JD and Kaufman RJ: Endoplasmic reticulum stress and oxidative stress: a vicious cycle or a double-edged sword? Antioxid Redox Signal 9: 2277-2293, 2007.

46. Salazar M, Carracedo A, Salanueva IJ, Hernández-Tiedra S, Egia A, Lorente M, Vázquez P, Torres S, Iovanna JL, Guzmán M, et al: TRB3 links ER stress to autophagy in cannabinoid antitumoral action. Autophagy 5: 1048-1049, 2009.

47. Pellerito O, Calvaruso G, Portanova P, De Blasio A, Santulli A, Vento R, Tesoriere G and Giuliano M: The synthetic cannabinoid WIN 55,212-2 sensitizes hepatocellular carcinoma cells to tumor necrosis factor-related apoptosis-inducing ligand (TRAIL)induced apoptosis by activating p8/CCAAT/enhancer binding protein homologous protein $(\mathrm{CHOP}) /$ death receptor 5 (DR5) axis. Mol Pharmacol 77: 854-863, 2010.

48. Galve-Roperh I, Sanchez C, Cortes ML, del Pulgar TG, Izquierdo $M$ and Guzman M: Anti-tumoral action of cannabinoids: involvement of sustained ceramide accumulation and extracellular signal-regulated kinase activation. Nat Med 6: 313-319, 2000 
49. Luca T, Di Benedetto G, Scuderi MR, Palumbo M, Clementi S, Bernardini R and Cantarella G: The CB1/CB2 receptor agonist WIN-55,212-2 reduces viability of human Kaposi's sarcoma cells in vitro. Eur J Pharmacol 616: 16-21, 2009.

50. Park JM, Xian XS, Choi MG, Park H, Cho YK, Lee IS, Kim SW and Chung IS: Antiproliferative mechanism of a cannabinoid agonist by cell cycle arrest in human gastric cancer cells. J Cell Biochem 112: 1192-1205, 2011.

51. Piñeiro R, Maffucci $T$ and Falasca M: The putative cannabinoid receptor GPR55 defines a novel autocrine loop in cancer cell proliferation. Oncogene 30: 142-152, 2011.

52. Hers I, Vincent EE and Tavaré JM: Akt signalling in health and disease. Cell Signal 23: 1515-1527, 2011.

53. Cudaback E, Marrs W, Moeller T and Stella N: The expression level of $\mathrm{CB} 1$ and $\mathrm{CB} 2$ receptors determines their efficacy at inducing apoptosis in astrocytomas. PLoS One 5: e8702, 2010.

54. Salazar M, Carracedo A, Salanueva IJ, Hernández-Tiedra S, Lorente M, Egia A, Vázquez P, Blázquez C, Torres S, García S, et al: Cannabinoid action induces autophagy-mediated cell death through stimulation of ER stress in human glioma cells. J Clin Invest 119: 1359-1372, 2009.

55. Torres S, Lorente M, Rodríguez-Fornés F, Hernández-Tiedra S, Salazar M, García-Taboada E, Barcia J, Guzmán M and Velasco G: A combined preclinical therapy of cannabinoids and temozolomide against glioma. Mol Cancer Ther 10: 90-103, 2011.
56. Vara D, Salazar M, Olea-Herrero N, Guzmán M, Velasco G and Díaz-Laviada I: Anti-tumoral action of cannabinoids on hepatocellular carcinoma: role of AMPK-dependent activation of autophagy. Cell Death Differ 18: 1099-1111, 2011.

57. Shrivastava A, Kuzontkoski PM, Groopman JE and Prasad A: Cannabidiol induces programmed cell death in breast cancer cells by coordinating the cross-talk between apoptosis and autophagy. Mol Cancer Ther 10: 1161-1172, 2011.

58. Wasik AM, Almestrand S, Wang X, Hultenby K, Dackland ÅL, Andersson P, Kimby E, Christensson B and Sander B: WIN55,212-2 induces cytoplasmic vacuolation in apoptosisresistant MCL cells. Cell Death Dis 2: e225, 2011.

59. Donadelli M, Dando I, Zaniboni T, Costanzo C, Dalla Pozza E, Scupoli MT, Scarpa A, Zappavigna S, Marra M, Abbruzzese A, et al: Gemcitabine/cannabinoid combination triggers autophagy in pancreatic cancer cells through a ROS-mediated mechanism. Cell Death Dis 2: e152, 2011.

60. Gustafsson SB, Lindgren T, Jonsson M and Jacobsson SO: Cannabinoid receptor-independent cytotoxic effects of cannabinoids in human colorectal carcinoma cells: synergism with 5-fluorouracil. Cancer Chemother Pharmacol 63: 691-701, 2009. 